\title{
SPECTRUM OF THE LAPLACIAN ON A MANIFOLD OF NEGATIVE CURVATURE. II
}

\author{
MARK A. PINSKY
}

\section{Introduction}

In the previous paper we determined upper and lower bounds for the quantity

$$
\lambda_{1}=\inf _{f \neq 0} \frac{\int_{M}|d f|^{2}}{\int_{M}|f|^{2}},
$$

the infimum of the $L^{2}$-spectrum of the Laplacian on a class of Riemannian manifolds which includes all simply connected complete two-dimensional manifolds of non positive curvature. This problem has been discussed by several authors [2]-[8]. In this paper we investigate the nature of the generalized eigenfunctions.

It is well known that in the classical case of the Lobaschevsky space of constant negative curvature $\left(=-k^{2}\right)$, the generalized eigenfunctions can be computed explicitly. For example, the differential equation for rotationally invariant eigenfunctions is

$$
f^{\prime \prime}+k \operatorname{coth} k r f^{\prime}+\lambda f=0\left(\lambda \geqslant \frac{k^{2}}{4}\right) .
$$

The solution of this equation which remains regular at $r=0$ is the Legendre function

$$
\begin{aligned}
f & =P_{\nu}(\cosh k r)\left(\nu=-\frac{1}{2}+i \sqrt{\lambda / k^{2}-1 / 4}\right) \\
& =\frac{1}{\pi} \int_{0}^{\pi}(\cosh k r+\sinh k r \cos \varphi)^{\nu} d \varphi .
\end{aligned}
$$

When $r \rightarrow \infty, P_{\nu}$ has the asymptotic behavior

$$
\left|P_{\nu}(\cosh k r)\right| \sim e^{-k r / 2}(r \rightarrow \infty),
$$

which implies that $f$ is not square integrable with respect to the Riemannian volume element $\sinh k r d r$. Hence the Laplacian has a purely continuous spectrum.

Received, February 8, 1978, and in revised form, June 30, 1978. Supported by National Science Foundation Grant MCS 78-02144 
When we pass to a manifold of variable negative curvature, the following quantity plays a role:

$$
\lambda_{1}^{*}=\frac{1}{4} \inf _{\theta} \lim _{r \rightarrow \infty}|K(r, \theta)|,
$$

where $K(r, \theta)$ is the Gaussian curvature in geodesic polar coordinates. The main theorem states that, if the above limit is attained with sufficient rapidity, then for $\lambda>\lambda_{1}^{*}$ there are no square integrable eigenfunctions. From this theorem we obtain the result that if $r \rightarrow|K(r, \theta)|$ is monotone decreasing, then the spectrum is purely continuous (Recent work of Ben-Artzi shows that in many cases this can be strengthened to absolutely continuous). Examples show that if the monotonicity condition is violated, then there may exist an (arbitrarily large) finite number of square-integrable eigenfunctions.

It is a pleasure to acknowledge helpful conversations with J. Rauch and M. Ben-Artzi.

\section{Statement of the problem}

The metric in geodesic polar coordinates has the form

$$
d s^{2}=d r^{2}+G(r, \theta)^{2} d \theta^{2}
$$

where

$$
G\left(0^{+}, \theta\right)=0, \quad G_{r}\left(0^{+}, \theta\right)=1, \quad \frac{G_{r r}(r, \theta)}{G(r, \theta)}=-K(r, \theta) \geqslant 0,
$$

and the subscripts denote partial derivatives. From the initial conditions, it is clear that $\left(G_{r} / G\right) \sim 1 / r$ when $r \rightarrow 0$. The curvature function $K(r, \theta)$ is assumed piecewise continuous. The Laplacian has the form

$$
-\Delta f=f_{r r}+G^{-1} G_{r} f_{r}+G^{-2} f_{\theta \theta}-G^{-3} G_{\theta} f_{\theta} .
$$

$\Delta$ is a symmetric, nonnegative operator in the Hilbert space $L^{2}(M)$, where the inner product is defined by

$$
\left(f_{1}, f_{2}\right) \equiv \int_{0}^{\infty} \int_{0}^{2 \pi} f_{1}(r, \theta) f_{2}(r, \theta) G(r, \theta) d r d \theta
$$

We say that $\lambda$ is an eigenvalue of $\Delta$ if there exists $f \in L_{2}(M)$ satisfying the equation $\Delta f=\lambda f$ in the classical sense. The bound of the $L^{2}$-spectrum is defined by

$$
\lambda_{1}=\inf _{f \neq 0} \frac{\int_{0}^{\infty} \int_{0}^{2 \pi}\left[f_{r}^{2}+f_{\theta}^{2} / G^{2}\right] G d r d \theta}{\int_{0}^{\infty} \int_{0}^{2 \pi} f^{2} G d r d \theta}
$$


where the infimum is taken over all $f \in L^{2}$ with piecewise smooth derivatives. Clearly any eigenvalue $\lambda$ satisfies the inequality $\lambda \geqslant \lambda_{1}$. Let us define $\mu \geqslant 0$ as the pointwise infimum

$$
\mu=\inf _{\substack{r>0 \\ 0<\theta<2 \pi}} \frac{G_{r}}{G} .
$$

It was shown in [8] that $\lambda_{1} \geqslant \mu^{2} / 4$. Therefore any eigenvalue must satisfy $\lambda \geqslant \mu^{2} / 4$.

Theorem 1. $\mu^{2} / 4$ is not an eigenvalue of $\Delta$.

Proof. Let $h(r, \theta) \equiv G_{r}-\mu G \geqslant 0$. Thus for any $f$

$$
\mu \int_{0}^{\infty} f^{2} G d r+\int_{0}^{\infty} h(r, \theta) f^{2} d r=\int_{0}^{\infty} G_{r} f^{2} d r .
$$

By Schwarz's inequality,

$$
\int_{0}^{\infty} G_{r} f^{2} d r=-2 \int_{0}^{\infty} G f f_{r} d r \leqslant 2\left(\int_{0}^{\infty} G f^{2} d r\right)^{1 / 2}\left(\int_{0}^{\infty} G f_{r}^{2} d r\right)^{1 / 2} .
$$

Dividing the resulting inequality by $\left(\int_{0}^{\infty} f^{2} G d r\right)^{1 / 2}$, we have

$$
I+\mu\left(\int_{0}^{\infty} f^{2} G d r\right)^{1 / 2} \leqslant 2\left(\int_{0}^{\infty} f_{r}^{2} G d r\right)^{1 / 2},
$$

where

$$
I \equiv \int_{0}^{\infty} h(r, \theta) f^{2} d r /\left(\int_{0}^{\infty} f^{2} G d r\right)^{1 / 2} \geqslant 0 .
$$

Squaring the result gives

$$
I^{2}+2 \mu I\left(\int_{0}^{\infty} f^{2} G d r\right)^{1 / 2}+\mu^{2} \int_{0}^{\infty} f^{2} G d r \leqslant 4 \int_{0}^{\infty} f_{r}^{2} G d r
$$

Integrating this on $[0,2 \pi]$ and adding in the angular term, we obtain

$$
\begin{gathered}
\int_{0}^{2 \pi}\left[I^{2}+2 \mu I\left(\int_{0}^{\infty} f^{2} G d r\right)^{1 / 2}\right] d \theta+\mu^{2} \int_{0}^{\infty} \int_{0}^{2 \pi} f^{2} G d r d \theta \\
\leqslant 4 \int_{0}^{\infty} \int_{0}^{2 \pi}\left(f_{r}^{2}+\frac{f_{\theta}^{2}}{G^{2}}\right) G d r d \theta
\end{gathered}
$$

Now let $f$ be an eigenfunction with eigenvalue $=\mu^{2} / 4$. Then the right-hand side of $(1.1)=\mu^{2} \int_{0}^{\infty} \int_{0}^{2 \pi} \mathrm{f}^{2} G d r d \theta$, and thus

$$
\int_{0}^{2 \pi}\left(I^{2}+2 \mu I\left(\int_{0}^{\infty} f^{2} G d r\right)^{1 / 2}\right) d \theta \leqslant 0 .
$$

Hence $I=0$ which means that $h=0$ and hence $G_{r}=\mu G$, which contradicts $G_{r} / G \sim 1 / r$ when $r \rightarrow 0$. 


\section{The rotationally symmetric case}

Let $G(r)$ be the density of the metric tensor in a system of geodesic polar coordinates. We make the following hypotheses:

$$
\begin{gathered}
G(0)=0, G^{\prime}(0)=1, G^{\prime \prime}(r) \geqslant 0, \\
\frac{G^{\prime \prime}}{G}=k^{2}+\varepsilon(r)\left(r \geqslant r_{0}\right),
\end{gathered}
$$

where

$$
\varepsilon(r) \geqslant 0, \int_{r_{0}}^{\infty} \varepsilon(r) d r<\infty .
$$

Define the associated potential function by the formula

$$
V(r)=\frac{1}{2} \frac{G^{\prime \prime}}{G}-\frac{1}{4}\left[\frac{G^{\prime}}{G}\right]^{2}
$$

Lemma 2.1. $\left|G^{\prime} / G-k\right| \leqslant \varepsilon_{1}(r)$ where $\varepsilon_{1}(r) \geqslant 0, \int_{r_{0}}^{\infty} \varepsilon_{1}(r) d r<\infty$.

Proof. Let $H=G^{\prime} / G$. Then $H$ satisfies the differential equation

$$
H^{\prime}+H^{2}=k^{2}+\varepsilon(r)\left(r \geqslant r_{0}\right) .
$$

By a previous result [7] we know that $\lim _{r \rightarrow \infty} H(r)=k$. We consider two cases.

Case 1. $H\left(r_{1}\right)>k$ for some $r_{1}>r_{0}$.

In this case let $H_{1}$ be the solution of $H_{1}^{\prime}+H_{1}^{2}=k^{2}, H_{1}\left(r_{1}\right)=H\left(r_{1}\right)$. Then

$$
\begin{aligned}
& \left(H-H_{1}\right)^{\prime}+\left(H-H_{1}\right)\left(H+H_{1}\right)=\varepsilon(r) \geqslant 0\left(r \geqslant r_{1}\right), \\
& \left(H-H_{1}\right)\left(r_{1}\right)=0 .
\end{aligned}
$$

Therefore

$$
\left(H-H_{1}\right)(r)=\int_{r_{1}}^{r} \varepsilon(s) \exp \left\{\int_{r}^{s}\left(H+H_{1}\right)(u) d u\right\} d s \geqslant 0 .
$$

But $H_{1}$ can be explicitly computed:

$$
\begin{aligned}
H_{1}(r) & =k \frac{1+C e^{-2 k r}}{1-C e^{-2 k r}}\left(C e^{-2 k r_{1}} \equiv \frac{H\left(r_{1}\right)-k}{H\left(r_{1}\right)+k}>0\right), \\
& >k .
\end{aligned}
$$

Thus $H(r)>k$ for all $r>r_{1}$. Since $\varepsilon-H^{\prime}=H^{2}-k^{2}>0$, we have

$$
H=k\left[1+\frac{\varepsilon-H^{\prime}}{k^{2}}\right]^{1 / 2} \leqslant k\left[1+\frac{\varepsilon-H^{\prime}}{2 k^{2}}\right] .
$$

Define $\varepsilon_{1}(r)=\left(\varepsilon-H^{\prime}\right) /(2 k)$. Then $\varepsilon_{1}(r) \geqslant 0$ and $\int_{r_{0}}^{\infty} \varepsilon_{1}(r) d r<\infty$. Therefore we have proved that $k \leqslant H(r) \leqslant k+\varepsilon_{1}(r)$, as required. 
Case 2. $H(r) \leqslant k$ for all $r>r_{0}$.

In this case, we have $H^{\prime}-\varepsilon=k^{2}-H^{2} \geqslant 0$. As before

$$
H=k\left[1-\frac{H^{\prime}-\varepsilon}{k^{2}}\right]^{1 / 2} \geqslant k\left[1-\frac{H^{\prime}-\varepsilon}{2 k^{2}}\right] .
$$

In this case we set $\varepsilon_{1}(r)=\left(H^{\prime}-\varepsilon\right) /(2 k)$. Then $\varepsilon_{1}(r) \geqslant 0$ and $\int_{r_{0}}^{\infty} \varepsilon_{1}(r) d r<$ $\infty$. Therefore we have proved that $k-\varepsilon_{1}(r) \leqslant H \leqslant k$ as required.

Lemma 2.2. $\left|V(r)-k^{2} / 4\right| \leqslant \varepsilon_{2}(r)$ where $\varepsilon_{2}(r) \geqslant 0, \int_{r_{0}}^{\infty} \varepsilon_{2}(r) d r<\infty$.

Proof. Using hypothesis (2.2) and definition (2.4) with $G^{\prime} / G=k+$ $\left(G^{\prime} / G-k\right)$, we obtain

$$
V(r)-\frac{k^{2}}{4}=\frac{1}{2} \varepsilon(r)-\frac{1}{2} k\left(\frac{G^{\prime}}{G}-k\right)+\frac{1}{4}\left(\frac{G^{\prime}}{G}-k\right)^{2} .
$$

But Lemma (2.2) states that $\left|G^{\prime} / G-k\right| \leqslant \varepsilon_{1}(r)$. Therefore each of the three terms is integrable on $\left[r_{0}, \infty\right)$, hence the result.

Let $f$ be an eigenfunction satisfying the differential equation

$$
f^{\prime \prime}+\frac{G^{\prime}}{G} f+\lambda f=0 .
$$

Lemma 2.3. $F=G^{1 / 2} f$ satisfies the differential equation

$$
F^{\prime \prime}+\lambda F-V F=0 .
$$

Proof. Write $f=F \cdot G^{-1 / 2}$. Then

$$
\begin{aligned}
& f^{\prime}=F^{\prime} G^{-1 / 2}-\frac{1}{2} G^{-3 / 2} G^{\prime} F \\
& f^{\prime \prime}=F^{\prime \prime} G^{-1 / 2}-G^{-3 / 2} G^{\prime} F^{\prime}+F\left\{\frac{3}{4} G^{-3 / 2}\left(G^{\prime}\right)^{2}-\frac{1}{2} G^{-3 / 2} G^{\prime \prime}\right\} .
\end{aligned}
$$

Therefore

$$
f^{\prime \prime}+\frac{G^{\prime}}{G} f^{\prime}=F^{\prime \prime} G^{-1 / 2}-F G^{-1 / 2} V(r),
$$

from which the equation follows.

Lemma 2.4. If $F \in L^{2}\left(r_{0}, \infty\right)$, then $F^{\prime} \in L^{2}\left(r_{0}, \infty\right)$.

Proof. From the differential equation (2.6), we see that $F^{\prime \prime} \in L^{2}\left(r_{0}, \infty\right)$. To obtain the stated conclusion extend $F$ to $(-\infty, \infty)$ so that $F \in C^{2} \cap L^{2}(-$ $\infty, \infty)$ and $F(r) \equiv 0$ for $r \leqslant-1$. Then $F^{\prime \prime} \in L^{2}(-\infty, \infty)$. In the domain of Fourier transforms, we now have

$$
|\xi \hat{F}(\xi)|^{2} \leqslant\left(1+|\xi|^{4} \mid\right)|\hat{F}(\xi)|^{2} .
$$

By construction, the right-hand member of the above equation is integrable by Parseval's theorem. Therefore $\xi \hat{F}(\xi) \in L^{2}(-\infty, \infty)$, which proves that $F^{\prime} \in L^{2}(-\infty, \infty)$.

Lemma 2.5. Let $f$ be a solution of (2.5) with $\lambda>k^{2} / 4, \int_{0}^{\infty} f(r)^{2} G(r) d r<$ $\infty$. Then $f \equiv 0$. 
Proof. Let $f$ be not identically zero, and let $A(r)=F^{\prime}(r)^{2}+\nu^{2} F(r)^{2}$, where $\nu^{2}=\lambda-k^{2} / 4$ and $F=G^{1 / 2} f$. Then $A(r)>0$ for all $r$ and, in consequence of (2.6),

$$
A^{\prime}(r)=2 F F^{\prime}\left\{V-k^{2} / 4\right\}
$$

Hence

$$
\frac{A^{\prime}(r)}{A(r)}=\frac{2 F F^{\prime}}{F^{\prime 2}+\nu^{2} F^{2}} \geqslant-\varepsilon_{2}(r) C(\nu),
$$

where we have used Lemma (2.2) and the inequality $\left(a^{2}+b^{2}\right) /\left(a^{2}+\nu^{2} b^{2}\right) \leqslant$ $\min \left(1, \nu^{-2}\right) \equiv C(\nu)$. Integrating the inequality from $r_{0}$ to $r$, we have that

$$
\log A(r)-\log A\left(r_{0}\right) \geqslant-C(\nu) \int_{r_{0}}^{r} \varepsilon_{2}(s) d s \geqslant-C(\nu) \int_{r_{0}}^{\infty} \varepsilon_{2}(s) d s,
$$

so that

$$
A(r) \geqslant A\left(r_{0}\right) \exp \left\{-C(\nu) \int_{r_{0}}^{\infty} \varepsilon_{2}(s) d s\right\} .
$$

Thus we have proved that

$$
F^{\prime}(r)^{2}+\nu^{2} F(r)^{2} \geqslant C_{3} .
$$

But Lemma (2.4) states that $\int_{r_{0}}^{\infty} F^{\prime}(r)^{2} d r<\infty$. Therefore $\int_{r_{0}}^{\infty}\left[F^{\prime}(r)^{2}+\right.$ $\left.\nu^{2} F(r)^{2}\right] d r<\infty$. This contradicts the inequality (2.7), and hence $f$ is identically zero.

We now consider eigenfunctions which depend on $\theta$. Let

$$
\begin{gathered}
f^{\prime \prime}+\frac{G^{\prime}}{G} f+\frac{1}{G^{2}} f_{\theta \theta}+\lambda f=0, \\
f_{n}(\bar{r}) \equiv \int_{0}^{2 \pi} f(r, \theta) e^{-i n \theta} d \theta, \\
F_{n}(r) \equiv f_{n}(r) G^{1 / 2}
\end{gathered}
$$

By Parseval's theorem, $\sum_{-\infty}^{\infty}\left|f_{n}(r)\right|^{2}=(1 / 2 \pi) \int_{0}^{2 \pi} f(r, \theta)^{2} d \theta$. Hence whenever $f \in L^{2}(M)$ it follows that $\int_{0}^{\infty} f_{n}(r)^{2} G(r) d r<\infty$. Furthermore, $f_{n}$ satisfies the differential equation

$$
f_{n}^{\prime \prime}+\frac{G^{\prime}}{G} f_{n}^{\prime}-\frac{n^{2}}{G^{2}} f_{n}+\lambda f_{n}=0,
$$

and by a direct calculation

$$
F_{n}^{\prime \prime}+\left(\lambda-\frac{n^{2}}{G^{2}}-V\right) F_{n}=0 .
$$

Now if $F_{n} \in L^{2}\left(r_{0}, \infty\right)$, the proof of Lemma (2.4) can be repeated to show that $F_{n}^{\prime} \in L^{2}\left(r_{0}, \infty\right)$. To finish the proof, we let

$$
A_{n}(r)=F_{n}^{\prime 2}+\nu^{2} F_{n}^{2}
$$


Then

$$
A_{n}^{\prime}=2 F_{n} F_{n}^{\prime}\left\{V-\frac{k^{2}}{4}+\frac{n^{2}}{G^{2}}\right\} .
$$

The term $n^{2} / G^{2}$ is clearly integrable on $\left(r_{0}, \infty\right)$. To see this, note from Lemma (2.1) that $G^{\prime} / G \geqslant k / 2$ for $r \geqslant r_{2}$. Then $G(r) \geqslant G\left(r_{2}\right) e^{k r / 2}$ hence $n^{2} / G^{2}=O\left(e^{-k r}\right)$. Thus

$$
\begin{aligned}
\frac{A_{n}^{\prime}}{A_{n}} & \geqslant-\left[\varepsilon_{2}(r)+O\left(e^{-k r}\right)\right] C(\nu), \\
A_{n}(r) & \geqslant A_{n}\left(r_{0}\right) C_{4}\left(r \geqslant r_{0}\right),
\end{aligned}
$$

and again it follows that

$$
\int_{r_{0}}^{\infty}\left[F_{n}^{\prime}(r)^{2}+\nu^{2} F_{n}(r)^{2}\right] d r=\infty
$$

which yields a contradiction. Hence we have prove the following theorem.

Theorem 2. Let $G(r)$ define a rotationally invariant metric of nonpositive curvature, satisfying (2.1)-(2.3). Let $f \in L^{2}$ be an eigenfunction of $\Delta$ with $\lambda>k^{2} / 4$. Then $f$ is identically zero.

Corollary. Assume in addition that (2.2) is satisfied for all $r>0$, where $\varepsilon(r)$ satisfies (2.3). Then the Laplacian has a purely continuous spectrum.

Proof. In this case we know from McKean's result [5] that the spectrum of $\Delta$ is contained in $\left[k^{2} / 4, \infty\right]$. By the above theorem, the open interval $\left(k^{2} / 4, \infty\right)$ contains no eigenvalues. To show that $k^{2} / 4$ is not an eigenvalue, we will apply Theorem 1 , for which we must show that $\inf _{r>0}\left(G^{\prime} / G\right)=k$. To see this, recall [7] that $G^{\prime \prime} / G \geqslant k^{2}$ everywhere implies that $G^{\prime} / G \geqslant k$ coth $k r \geqslant k$. But (2.2) implies that $\lim _{r \rightarrow \infty}\left(G^{\prime} / G\right)=k$. Therefore $\inf _{r>0}\left(G^{\prime} / G\right)=k$ which was to be shown.

Example. The following example illustrates the possibility of eigenvalues with $\lambda<\lambda_{1}^{*}$. Let the metric be defined by

$$
\begin{array}{rlrl}
G(r) & =r, & & 0 \leqslant r \leqslant R, \\
& =R \frac{\cosh ^{2} k\left(r-r_{0}\right)}{\cosh ^{2} k\left(R-r_{0}\right)}, & r \geqslant R,
\end{array}
$$

where $2 k R>1$, and $r_{0}$ is chosen so that $2 k \tanh k\left(R-r_{0}\right)=1 / R$. This ensures that $G$ and $G^{\prime}$ are continuous on $[0, \infty)$. A short calculation shows that

$$
\begin{gathered}
\frac{G^{\prime}}{G}=2 k \tanh k\left(r-r_{0}\right)(r>R), \\
-K=\frac{G^{\prime \prime}}{G}=4 k^{2} \tanh ^{2} k\left(r-r_{0}\right)+2 k^{2} \operatorname{sech}^{2} k\left(r-r_{0}\right)(r>R) .
\end{gathered}
$$


The latter quantity is nonnegative and tends to $4 k^{2}$ when $r \rightarrow \infty$.

The eigenvalue equation takes the following form

$$
\begin{aligned}
& f^{\prime \prime}+\frac{1}{r} f^{\prime}+\lambda f=0, \quad r \leqslant R, \\
& f^{\prime \prime}+2 k \tanh k\left(r-r_{0}\right) f^{\prime}+\lambda f=0, \quad r>R .
\end{aligned}
$$

The regular solution for $r<R$ is $f=J_{0}(r \sqrt{\lambda})$. For $r>R$ we rewrite the equation in the form

$$
\left[\cosh k\left(r-r_{0}\right) f\right]^{\prime \prime}+\left(\lambda+k^{2}\right)\left[\cosh k\left(r-r_{0}\right) f\right]=0 .
$$

For $\lambda<k^{2}$, the $L^{2}$ solution is

$$
f=\frac{A}{\cosh k\left(r-r_{0}\right)} \exp (-r \tau)
$$

where $\tau=\sqrt{k^{2}-\lambda}$. To satisfy the continuity of $f$ and $f^{\prime}$, we must have

$$
\begin{aligned}
J_{0}(R \sqrt{\lambda})=\frac{A}{\cosh k\left(R-r_{0}\right)} \exp (-R \tau), \\
\sqrt{\lambda} J_{0}^{\prime}(R \sqrt{\lambda})=\frac{A}{\cosh ^{2} k\left(R-r_{0}\right)}\left[-\cosh k\left(R-r_{0}\right) \tau \exp (-R \tau)\right. \\
\left.\quad-k \sinh k\left(R-r_{0}\right) \exp (-R \tau)\right] .
\end{aligned}
$$

Dividing these and simplifying, we have

$$
\frac{-\theta J_{0}^{\prime}(\theta)}{J_{0}(\theta)}=\frac{1}{2}+\sqrt{R^{2} k^{2}-\theta^{2}},
$$

where we have set $\theta=R \sqrt{\lambda}$ and used the fact that $2 k R \tanh k\left(R-r_{0}\right)=1$. This equation can be solved graphically in terms of the roots of $J_{0}$. Indeed, let $\theta_{1}<\theta_{2}<\cdots$ be the positive roots of $J_{0}(\theta)=0$. If we compare the graph of $\theta \rightarrow \theta J_{0}^{\prime}(\theta) / J_{0}(\theta)$ with the graph of $\theta \rightarrow \frac{1}{2}+\left(R^{2} k^{2}-\theta^{2}\right)^{\frac{1}{2}}$, it is clear that if $\boldsymbol{\theta}_{n}<k R$, the above transcendental equation has at least $n$ roots. Hence there exist $n$ eigenfunctions with eigenvalues in the interval $\left(0, \lambda_{1}^{*}\right)$.

\section{Improvement of the condition (2.3)}

We will show that the nonexistence of square integrable eigenfunctions can be obtained under more general conditions. Then conditions are closely related to some recent results of $M$. Ben-Artzi [1] on the spectrum of Schrodinger's equation.

To express these conditions, we let

$$
\mathrm{a}_{n}(t) \equiv V(t)-\frac{k^{2}}{4}+\frac{n^{2}}{G^{2}(t)},
$$


and require that

$$
\begin{aligned}
& q_{n}^{\prime} \in L^{1}\left(r_{0}, \infty\right), \\
& q_{n} \in L^{p}\left(r_{0}, \infty\right),
\end{aligned}
$$

for some $0<p<\infty$. Then we have the following result.

Theorem 3. Let $G(r)$ define a rotationally invariant metric of non-positive curvature, when $V(r)$ satisfies (3.1)-(3.3). Let $f \in L^{2}$ be an eigenfunction of $\Delta$ with $\lambda>k^{2} / 4$. Then $f$ is identically zero.

Proof. As before, let $F_{n}(r)=G^{1 / 2}(r) \int_{0}^{2 \pi} f(r, \theta) e^{-i n \theta} d \theta$. Then $F_{n}$ satisfies $F_{n}^{\prime \prime}+\left(\lambda-n^{2} / G^{2}-V\right) F_{n}=0$. Introduce "polar coordinates" by the equations

$$
\begin{gathered}
A_{n}(r) \equiv F_{n}^{\prime}(r)^{2}+\nu^{2} F_{n}(r)^{2}\left(\nu^{2}=\lambda-\frac{k^{2}}{4}\right) \\
\theta_{n}(r) \equiv-\tan ^{-1}\left[\frac{F_{n}^{\prime}(r)}{\nu F_{n}(r)}\right] .
\end{gathered}
$$

From the differential equation for eigenfunctions, we have

$$
\begin{gathered}
A_{n}^{\prime}=2 F_{n} F_{n}^{\prime} a_{n}, \\
\theta_{n}^{\prime}=\nu-\left(a_{n} \sin ^{2} \theta_{n}\right) / \nu .
\end{gathered}
$$

Therefore $A_{n}^{\prime}=\left(2 A_{n} \sin \theta_{n} \cos \theta_{n}\right) / \nu,\left|\theta_{n}^{\prime}-\nu\right| \leqslant \nu^{-1}\left|\mathfrak{q}_{n}\right|$. Finally, define

$$
\Phi_{n}(t) \equiv \nu \int_{0}^{t} \sin 2 \theta_{n}(s) d s .
$$

Lemma. For each $n=1,2, \cdots$ we have $M_{n} \equiv \sup _{t \geqslant 0}\left|\Phi_{n}(t)\right|<\infty$.

Proof. Define a new variable of integration by $u=\theta_{n}(s)$, valid for sufficiently large $s$. Then $d u=\nu\left[1-\bar{q}_{n}(u)\right] d s$, where $\bar{q}_{n}(u)=\left(q_{n}(s) \sin ^{2} \theta_{n}\right) / v^{2}$. Choose $t_{0}$ such that $\left|q_{n}(t)\right| \leqslant \nu^{2} / 2$ for $t \geqslant t_{0}$. Then we have

$$
\begin{aligned}
\Phi_{n}(t)-\Phi_{n}\left(t_{0}\right) & =\nu \int_{t_{0}}^{t} \sin \theta_{n}(s) d s=\int_{\theta_{n}\left(t_{0}\right)}^{\theta_{n}(t)} \frac{\sin 2 u}{1-\bar{q}_{n}(u)} d u \\
& =\sum_{k=0}^{m} I_{k}+I I_{m},
\end{aligned}
$$

where $m$ is an integer larger than $p$ and

$$
\begin{gathered}
I_{k}(t) \equiv \int_{\theta_{n}\left(t_{0}\right)}^{\theta_{n}(t)} \sin 2 u \bar{q}_{n}(u)^{k} d u, \\
I I_{m}(t) \equiv \int_{\theta_{n}\left(t_{0}\right)}^{\theta_{n}(t)} \sin 2 u \frac{\bar{q}_{n}(u)^{m+1}}{1-\bar{q}_{n}(u)} d u .
\end{gathered}
$$

We must show that each of these terms remains bounded when $t \rightarrow \infty$. For this purpose, let $\Psi_{k}(t) \equiv \int_{0}^{t} \sin 2 u \sin ^{2 k} u d u, k \leqslant m$. Then $\Psi_{k}$ is bounded 
when $t \rightarrow \infty$ and

$$
\begin{aligned}
I_{k}(t) & =\int_{\theta_{n}\left(t_{0}\right)}^{\theta_{n}(t)} q_{n}(s)^{k} \sin ^{2 k} u \sin 2 u d u=\int_{\theta_{n}\left(t_{0}\right)}^{\theta_{n}(t)} q_{n}(s)^{k} d \Psi_{k}(u) \\
& =\left.q_{n} \Psi_{k}\right|_{\theta_{n}\left(t_{0}\right)} ^{\theta_{n}\left({ }^{(t)}\right.}-k \int_{\theta_{n}\left(t_{0}\right)}^{\theta_{n}(t)} \frac{q_{n}(s)^{k-1} q_{n}^{\prime}(s) \Psi(u) d u}{v\left[1-\grave{q}_{n}(u)\right]}
\end{aligned}
$$

By hypothesis (3.2), the final integral remains bounded when $t \rightarrow \infty$. Therefore $I_{k}(t)$ remains bounded when $t \rightarrow \infty$. To analyze $I_{m}(t)$, we note that

$$
\left|I I_{m}(t)\right| \leqslant 2 \int_{\theta_{n}\left(t_{0}\right)}^{\theta_{n}(t)}\left|\bar{q}_{n}(u)\right|^{m+1} d u,
$$

which remains finite by hypothesis (3.3).

Proof of Theorem 3. From (3.6) we have

$$
\begin{aligned}
\log A_{n}(t)-\log A_{n}\left(t_{0}\right) & =\nu^{-1} \int_{t_{0}}^{t} \sin 2 \theta_{n}(s) q_{n}(s) d s \\
& =\nu^{-2} \int_{t_{0}}^{t} q_{n}(s) d \Phi_{n}(s) d s .
\end{aligned}
$$

The final integral is integrated by parts. From hypothesis (3.2) and the lemma, we see that the first terms remain bounded and the integral converges when $t \rightarrow \infty$. Therefore

$$
\lim _{t \rightarrow \infty} \log A_{n}(t)>-\infty .
$$

Hence $A_{n}(t) \geqslant C>0$, from which the conclusion follows as in the previous theorem.

\section{The general case}

We will show the nonexistence of eigenvalues for $\lambda>k^{2} / 4$, where $k$ is any limiting value of the curvature. For this purpose we can localize in a sector $\alpha \leqslant \theta \leqslant \beta, r_{0} \leqslant r<\infty$. The main hypothesis is that for some sector the metric is independent of $\theta$; we make a Fourier sine expansion of the eigenfunction, leading to a system of ordinary differential equations.

Let $f$ be an eigenfunction satisfying

$$
f_{r r}+\frac{G_{r}}{G} f_{r}+\frac{1}{G}\left(\frac{f_{\theta}}{G}\right)_{\theta}+\lambda f=0 .
$$

Let

$$
F_{n}(r) \equiv \int_{\alpha}^{\beta} f(r, \theta) G^{1 / 2}(r, \theta) \sin \left[\frac{n \pi(\theta-\alpha)}{\beta-\alpha}\right] d \theta .
$$

By Parseval's theorem, $\Sigma_{1}^{\infty} F_{n}(r)^{2}=\left(\int_{\alpha}^{\beta} f^{2} G d \theta\right)(\beta-\alpha) / 2$. 
Then $F_{n}$ satisfies the equation

$$
F_{n}^{\prime \prime}+\left(\lambda-\frac{k^{2}}{4}\right) F_{n}=I_{n}+I I_{n}
$$

where

$$
\begin{aligned}
& I_{n}=\int_{\alpha}^{\beta} f G^{1 / 2}\left(V-\frac{k^{2}}{4}\right) \sin \left[\frac{n \pi(\theta-\alpha)}{\beta-\alpha}\right] d \theta, \\
& I I_{n}=-\int_{\alpha}^{\beta} \frac{1}{G^{1 / 2}}\left(\frac{f_{\theta}}{G}\right)_{\theta} \sin \left[\frac{n \pi(\theta-\alpha)}{\beta-\theta}\right] d \theta .
\end{aligned}
$$

Now we assume specifically, that for some sector $\left[r_{0}, \infty\right) \times[\alpha, \beta]$ we have

$$
\begin{gathered}
G(r, \theta)=G(r), \\
\frac{G_{r r}}{G}=k^{2}+\varepsilon(r), \\
\varepsilon(r) \geqslant 0, \int_{r_{0}}^{\infty} \varepsilon(r) d r<\infty .
\end{gathered}
$$

Theorem 4. Under conditions (4.6)-(4.8), any square integrable solution of (4.1) with $\lambda>k^{2} / 4$ must be identically zero.

Proof. We proceed as in the rotationally invariant case. Defining $\nu^{2}=\lambda$ $-k^{2} / 4$ we simplify (4.3) to

$$
F_{n}^{\prime \prime}+\nu^{2} F_{n}=\varepsilon(r) F_{n}+\frac{n^{2} \pi^{2}}{G^{2}(\beta-\alpha)^{2}} F_{n}\left(r \geqslant r_{0}\right) .
$$

Defining $A_{n}=\left(F_{n}^{\prime}\right)^{2}+\nu^{2} F_{n}^{2}$, we have

$$
A_{n}^{\prime}=-2 F_{n}^{\prime} \varepsilon(r) F_{n}-\frac{2 n^{2} \pi^{2}}{(\beta-\alpha)^{2} G(r)^{2}} F_{n} F_{n}^{\prime}\left(r \geqslant r_{0}\right)
$$

and therefore

$$
\frac{A_{n}^{\prime}}{A_{n}} \geqslant-\left[\varepsilon(r)+\frac{n^{2} \pi^{2}}{(\beta-\alpha)^{2} G(r)^{2}}\right] C(\nu),
$$

where $C(\nu)=\sup \left(x_{1} x_{2} / x_{1}^{2}+\nu^{2} x_{2}^{2}\right)$. By hypotheses (4.7), (4.8) the quantity in brackets in (4.11) is integrable on $\left[r_{0}, \infty\right]$. Thus

$$
A_{n}(r) \geqslant A_{n}\left(r_{0}\right) \exp \left[-C \int_{r_{0}}^{\infty}\left[\varepsilon(r)+\frac{n^{2} \pi^{2}}{(\beta-\alpha)^{2} G(r)^{2}}\right] d r\right]>0 .
$$

Therefore either $A_{n}\left(r_{0}\right)=0$ or $\int_{r_{0}}^{\infty} F_{n}(r)^{2} d r=\infty$, and we have proved the theorem.

Added in Proof. Some extensions of these results have been obtained by H. Donnelly, Eigenvalues embedded in the continuum for negatively curved manifolds, Purdue University preprint. 


\section{References}

[1] M. Ben-Artzi, Eigenfunction expansions for ordinary differential operators, J. Math. Anal. Appl. 69 (1979) 304-314.

[2] S. Y. Cheng, Eigenvalue comparison theorems and their geometric applications, Math. Z. 143 (1975) 289-297.

[3] A. Debiard, B. Gaveau \& E. Mazet, Théorèmes de comparison en geomtrie Riemannianne, Publ. Res. Inst. Math. Sci. Kyoto 12 (1976) 391-425.

[4] P. Malliavin, Diffusions et geomtrie differentielle globale, Centro Math. Estivio, Varenna, 1975.

[5] H. P. McKean, An upper bound to the spectrum of $\Delta$ on a manifold of negative curvature, $\mathbf{J}$. Differential Geometry 4 (1970) 359-366.

[6] R. Osserman, Bonnesen-style isoperimetric inequalities, Amer. Math. Monthly 86 (1979) 1-29.

[7] M. Pinsky, An individual ergodic theorem for the diffusion on a manifold of negative curvature, Proc. Sympos. Stochastic Differential Equations, Academic Press, New York, 1977, 231-240.

[8] - The spectrum of the Laplacian on a manifold of negative curvature. I, J. Differential Geometry 13 (1978) 87-91.

NORTHWESTERN UNIVERSITY 